\title{
Intelligent Wireless Sensor Networks for Early Fire Warning System
}

\author{
Atıf Fazıl Önal iD, Burak Ülver (D), Alper Durusoy iD, Burcu Erkmen \\ Department of Electronics and Communication Engineering, YıldızTechnical University, İstanbul, Turkey
}

Cite this article as: Önal AF, Ülver B, Durusoy A, Erkmen B. Intelligent Wireless Sensor Networks for Early Fire Warning System. Electrica, vol. 19, DOI: 10.26650/electrica.2019.19019.

\begin{abstract}
Forest and wide area fires are one of the biggest obstacles to the protection of green areas, which are the life guarantee for future generations. These fires, which constitute a major threat to the ecosystem, can lead to human and animal deaths. In this work, an early warning system was proposed to detect forest fires with wireless sensor networks. The wireless sensor network consists of spatially distributed sensors to analyze the situations of the environment for various scenarios. Near real-time data from various sensors are transmitted to the server within mesh network structure via redundant paths using low cost and low power Zigbee. The communication in the system is continued thanks to this mesh design without any loss of data when there is a connection break between the sensor nodes and the beacons. Multi-layer feed forward neural networks are utilized for decision-making stage by adopting to the conditions of the environment rhythms. By designing a graphical user interface that works on a local machine, the approximate location of the modules, status color codes and status visualization with Google Maps on web server and notification of the relevant authorities are provided.
\end{abstract}

Keywords: Wireless sensor networks, forest fires, feed forward neural networks, warning system, web server

\section{Corresponding Author: \\ Burcu Erkmen}

\section{E-mail:}

bkapan@yildiz.edu.tr

Received: 29.03 .2019

Accepted: 19.07.2019

C Copyright 2019 by Electrica Available online at

http://electrica.istanbul.edu.tr

DOI: 10.26650/electrica.2019.19019

Content of this journal is licensed under a Creative Commons Attribution-NonCommercial 4.0 International License.

\section{Introduction}

Traditional methods such as local patrols, monitoring towers, aerial prevention, long-distance video detection and satellite tracking are used to detect early forest fires. It has been increased the interest in research cases in special areas such as providing real-time monitoring and early warning technology facilities of wireless sensor networks, fight against natural disasters and following the unattended ecological environment.

In the literature, distributed sensor nodes which used wireless sensor network protocols were investigated not only for early fire systems but also for abnormal status determinations as a result of meteorological natural hazard. ZigBee protocol based wireless sensor network was used for the detection of ultraviolet flames and forest fire in [1]. Wavelength detection of the flame has been carried out by using various electronic circuit elements such as ultrasonic photodetector and sensor nodes with low power consumption. In [2], authors performed in early forest fire detection system design and evaluations with wireless communication networks, the basic issues in the modeling of forest fires were introduced. Fire air index (FWI) system data were analyzed and different components that could be required for an effective fire detection system were investigated. The large areas of evaluation are divided into small areas called 'cells'. The 'k-coverage' method was used for the effective placement of cells. In [3], authors tried to provide near real-time detection of meteorological natural hazards, especially fire situations, with distributed wireless sensor networks. In the classification study, machine learning techniques have been applied to real-world events through data mining.

A wireless sensor network (WSN) for data harvesting to be used as raw input data into their control system is proposed in [4]. In this system, Fuzzy Logic algorithm is used for intelligent decision-making system for fire detection process which performs five membership functions such as temperature, smoke, light, humidity and distance. A cluster-based wireless sensor network 
paradigm for real-time detection of forest fire is proposed in [5]. In this paper, Ad hoc network related technology, the node hardware designing, the forest-fire forecasting model and the propagation characteristic of UHF wireless signals are discussed. In [6], the authors proposed to detect faulty nodes and calculate the probability of fire using Fuzzy logic. In [7], authors aim to identify firestorm in a forest and by predicting the firestorm in a forest the sensing ability of the sensor node. It is very difficult to detect the occurrence of fire with such systems of an insufficient number of sensor nodes. In addition, such systems with insufficient infrastructure cause delay in the alarm signal and have failed reporting. A Feed-forward Neural Network designed on FPGA was used in order to overcome such problems.

Soliman et al. [8] in their study, evaluated the situation of fire, low smoke level fire and high smoke level fire by combining wireless sensor networks with artificial neural networks. Chehri et al. [9] in their study monitored mines against fire by using wireless networks. Jain et al. [10] in their study placed in the form of clusters with equal spacing between the vertical and horizontal alignments. The information from the sensors is transmitted wirelessly from the node in the main hub to the server side. Early forest fire detection was tried to provide with wireless sensor network system via temperature, humidity and gas detector sensors in various literature researches [11, 12]. In [13], the high reliability and low cost system which is composed of wireless sensor network and GPRS network can realize repaid establishment of ad hoc networks of the sensor nodes as well as real-time collection, transmission and display of the information on various surrounding growth environment. Aslan et al. [14] in their work propose a comprehensive frame work for the use of wireless sensor networks for forest fire detection and monitoring. Their framework includes four main components of a wireless sensor network: the deployment scheme, the logical topology and architecture of the network, the intra-cluster communication scheme, and the inter-cluster communication scheme. Their system can provide both effective and efficient operation: consuming less energy without sacrificing the quick reaction capability. .In algorithmic manner, a bi-objective optimization model to seek the best locations for fire stations, accounting for access, service coverage and the impact of existing stations is proposed in [15].

Based on the literature, in order to establish such a system with minimum requirement, there has to be wireless sensor and communication networks including beacon and sensor nodes and local host. Number of sensor nodes should be increased not only to have wide coverage, but also to create redundant paths.

In our work, we propose an early warning system to detect forest fires with wireless sensor networks. Our motivation is to create scalable, modular, and user friendly an early fire warning system. System management is simpler because whole decision algorithm can be controlled remotely. The network consists of sensor nodes, beacons and server, which are distributed in the early forest fire detection process. Temperature, flame wavelength and air quality threshold values have been determined by the sensor nodes communicating with ZigBee wireless communication protocol. Within the scope of various scenarios, training and test data have been created according to these values. The training was carried out by selecting the artificial neural network architecture appropriate to the data set. Real-time data from the sensors is classified on the beacons and visualized to inform the end user.

The rest of this article is organized as follows. The next section provides a brief overview of wireless sensor networks and the IEEE ZigBee wireless communication protocol. Section 3, the sensor node, the beacon, and the overall system structure together with the relationship between these components. Section 4, feed forward neural network-based beacon design is implemented and the communication between the intermediate and the server is explained. Section 5, local server and server components are mentioned. Section 6 presents the simulations and results of this study. Finally, section 7 completes this study and presents future work.

\section{Wireless Sensor Networks for Fire Detection}

Wireless Sensor Networks are a set of tools that work independently from each other using sensors to cooperatively monitor physical or environmental conditions such as temperature, humidity, light, sound, pressure, vibration, object movements, pollution, soil composition, and noise level at the different location. A typical wireless sensor network is made up of hundreds or even thousands of sensor nodes connected by a wireless medium and exchanging information with each other. The sensor nodes, which are randomly distributed to large areas, consist of the beacon structure that collects, processes and extracts the data coming from other nodes, and finally the server part which we can visually observe these data. The mesh structure created in the study was carried out with ZigBee protocol with low cost and minimum energy consumption. The ZigBee protocol is easy to integrate into the system and thanks to its flexible structure, the routing of the message path can change dynamically in the network used to improve the performance of the application to be used. However, it has a lower data rate than the Bluetooth protocol and it has the disadvantage that data cannot be sent in large sizes.

In our system; temperature, air quality value and flame information are broadcast from the sensor nodes to the target Beacon addresses using the ZigBee protocol. These sensor values are classified with artificial neural network on the Raspberry Pi in the Beacon. This data is transmitted from the data storage server on Amazon Web Services (AWS), which acts as an intermediate server, to the server running locally.

\section{Mesh Topology and System Design}

The structure created in this study includes a server, two beacons and two sensor nodes connected to these beacons in order to create the infrastructure for the future study. In this topology, all sensor nodes shown in Figure 1 are connected to beacon and also connected to each other. Thus, if the connection between the sensor node and the Beacon breaks, the data continues to flow through the other sensor node. 


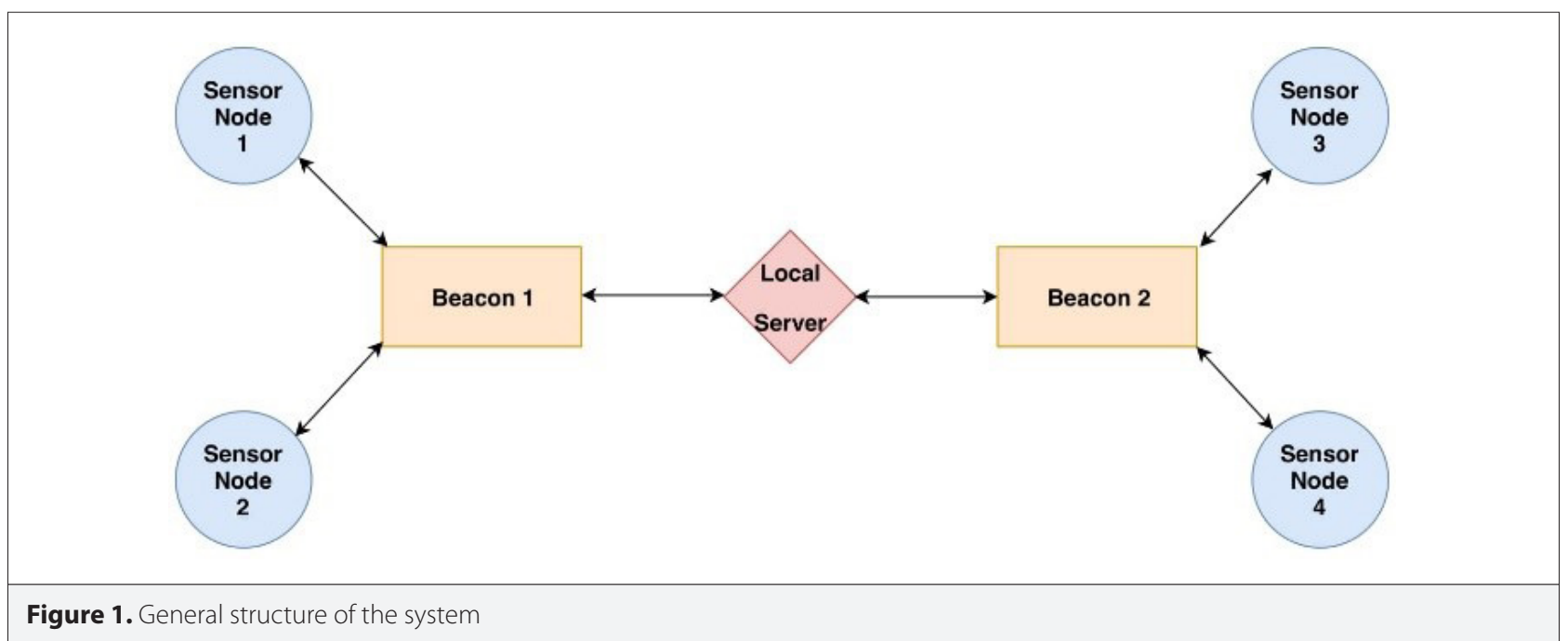

\section{Sensor nodes}

Fires change the temperature and humidity of the environment, but also CO (Carbon Monoxide) gas is released and causes the flame to form. The LM35 temperature sensor is used to detect the change of temperature at the sensor nodes as shown in Figure 2, the MQ-135 air quality sensor is used to measure the intensity of the smoke and the flame detector sensor is used to determine the presence of the flame. Because it forms the basis for future work, this study is limited to the number of 4 sensor nodes.

\section{Mesh topology}

Mesh topology in Wireless Sensor Networks has a structure that is resistant to line breaks due to providing redundancy. When a sensor node is in a state that cannot communicate with the beacon, data can be sent through other sensor nodes. In this study, each XBee wireless communication module, which operates the ZigBee protocol, is configured via the XCTU interface program. In this way, the sensor nodes and the beacon can

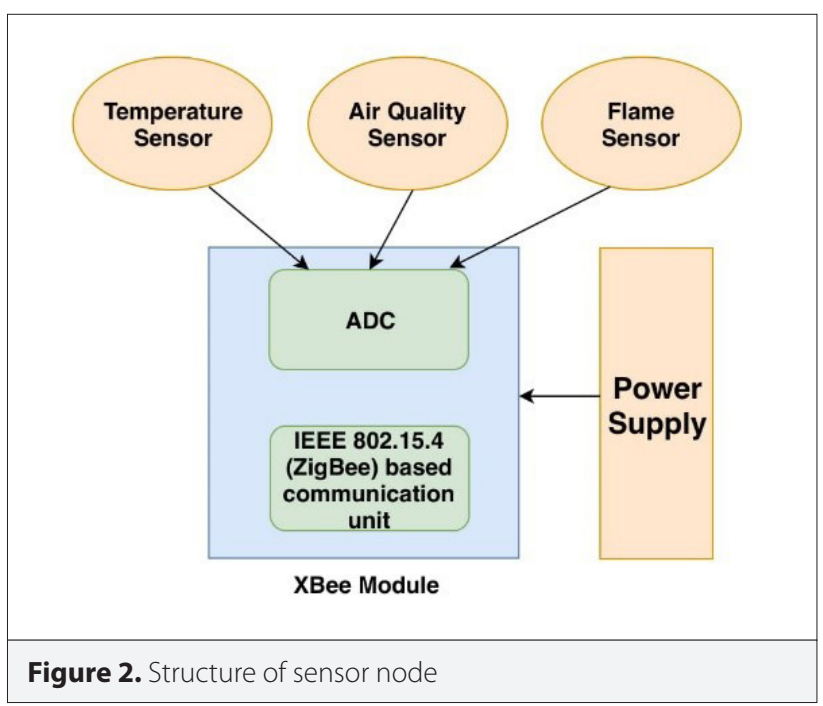

communicate using all the properties of the mesh structure as shown Figure 3.

\section{Beacon structure}

Beacon collects data from the sensors in the scanning area, collects data on the amount of gas and existence of flame, and then wirelessly puts it on the server. Beacon acts as a bridge between the sensors and the server. The Beacon has a receiver XBee module for collecting data from sensors in the structure. In this work, this module takes the data from the sensors and decides on the state of fire in the environment using the Multi-layer feed forward neural network structure run on the Raspberry Pi as shown in Figure 4. The data obtained from the artificial neural network output is transferred to the server side by using Wi-Fi module. The beacon can also access the Internet with a GPRS module integrated to Raspberry Pi.

\section{Intelligent Beacon Structure}

A data set was created to perform the classification process. First, the analog data generated by the sensors are converted to 10 -bit numerical values using the ADC unit of the XBee communication module. Numeric data is transmitted to the XBee
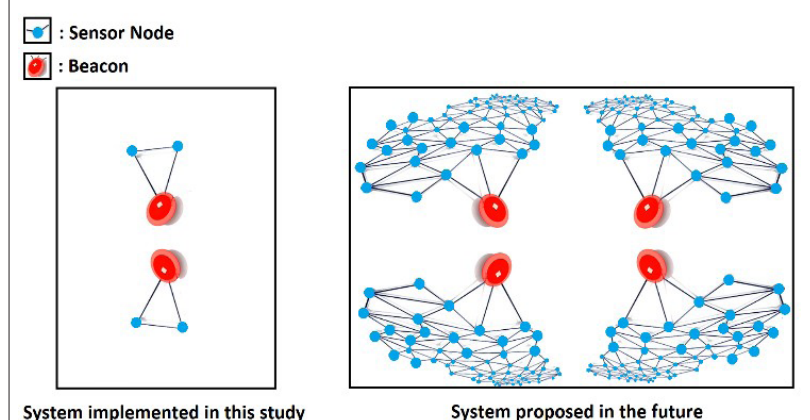

System proposed in the future

Figure 3. Illustration of sensor nodes and beacons in mesh structure 


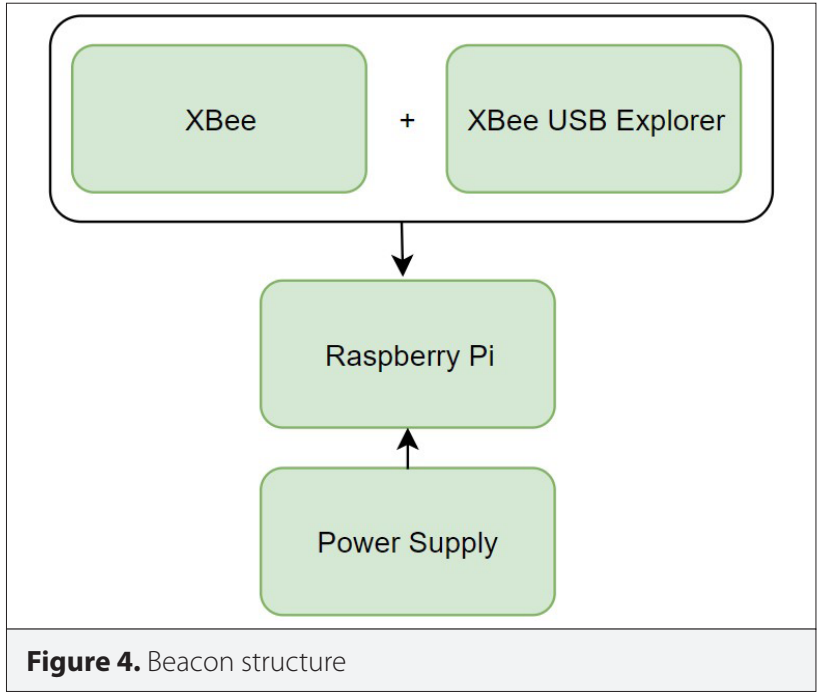

module in the Beacon with the XBee wireless communication module. Numerical values are transferred to the operating system running on Raspberry $\mathrm{Pi}$.

Table 1. The created dataset

\begin{tabular}{lcccc}
\hline $\begin{array}{l}\text { Range of } \\
\text { Temperature }\left({ }^{\circ} \mathrm{C}\right)\end{array}$ & $\begin{array}{c}\text { Air Quality } \\
\text { Sensor }\end{array}$ & $\begin{array}{c}\text { Flame } \\
\text { Sensor }\end{array}$ & $\begin{array}{c}\text { Number of } \\
\text { Samples }\end{array}$ & Label \\
\hline$(-20,40)$ & $0-500$ & 1 & 43000 & 1 \\
\hline$(40,60)$ & $0-500$ & 1 & 43000 & 2 \\
\hline$(60,120)$ & $500-700$ & 1 & 72000 & 1 \\
\hline$(40,60)$ & $500-700$ & 1 & 43000 & 2 \\
\hline$(60,120)$ & $0-500$ & 1 & 43000 & 2 \\
\hline$(60,120)$ & $500-700$ & 1 & 43000 & 2 \\
\hline$(-20,40)$ & $700-1023$ & 1 & 43000 & 2 \\
\hline$(40,60)$ & $700-1023$ & 1 & 43000 & 2 \\
\hline$(60,120)$ & $700-1023$ & 1 & 43000 & 2 \\
\hline$(-20,40)$ & $0-500$ & 0 & 2500000 & 0 \\
\hline$(40,60)$ & $0-500$ & 0 & 72000 & 1 \\
\hline$(-20,40)$ & $500-700$ & 0 & 72000 & 1 \\
\hline$(40,60)$ & $500-700$ & 0 & 72000 & 1 \\
\hline$(60,120)$ & $0-500$ & 0 & 72000 & 1 \\
\hline$(60,120)$ & $500-700$ & 0 & 43000 & 2 \\
\hline$(-20,40)$ & $700-1023$ & 0 & 72000 & 1 \\
\hline$(40,60)$ & $700-1023$ & 0 & 43000 & 2 \\
\hline$(60,120)$ & $00-1023$ & 0 & 43000 & 2 \\
\hline & & & & 2 \\
\hline
\end{tabular}

\section{Creating Dataset}

In this study, due to the low level of complexity, the data set including the values produced by the sensors to be used in the training were produced on the computer. According to the data from the sensors, the estimation will be based on 3 main scenarios. The conditions labeled as 0, 1 and 2 are listed below and created dataset is given in Table 1.

- Normal Situation (0): It is the case where the environment temperature is in the range of $(-20,40){ }^{\circ} \mathrm{C}$, the gas sensor ADC value is between 0 and 500 and the flame sensor produces the flame value. This is interpreted as normal.

- Suspicious Situation (1): It is the case where the ambient temperature is in the range of $(40,70){ }^{\circ} \mathrm{C}$, the gas sensor ADC measures between 500 and 700 ADC and the flame sensor produces the flame present value. In this case, only one sensor is enough to interpret as a suspicious condition if a value of a level above the normal level occurs.

- Critical Situation (2): It is the case where the temperature is above $70^{\circ} \mathrm{C}$, the gas sensor measures more than 700 ADC and the flame is present. If two or all of these three conditions are provided, then the events are interpreted as critical situations and the warning systems are executed

In total, the data size 3.405 .000 , training data has 3.370 .950 and test data has 34.050 examples.

\section{Architecture of Feed Forward Neural Network}

Designed Multi-Layer Feed Forward Neural Network architecture as shown in Figure 5 consists of an input layer, 2 hidden layers and output layer. The input layer has 3 input values, the first hidden layer has 12 neurons, the second hidden layer has 8 neurons and output layer has 3 neurons, ReLU is used as the

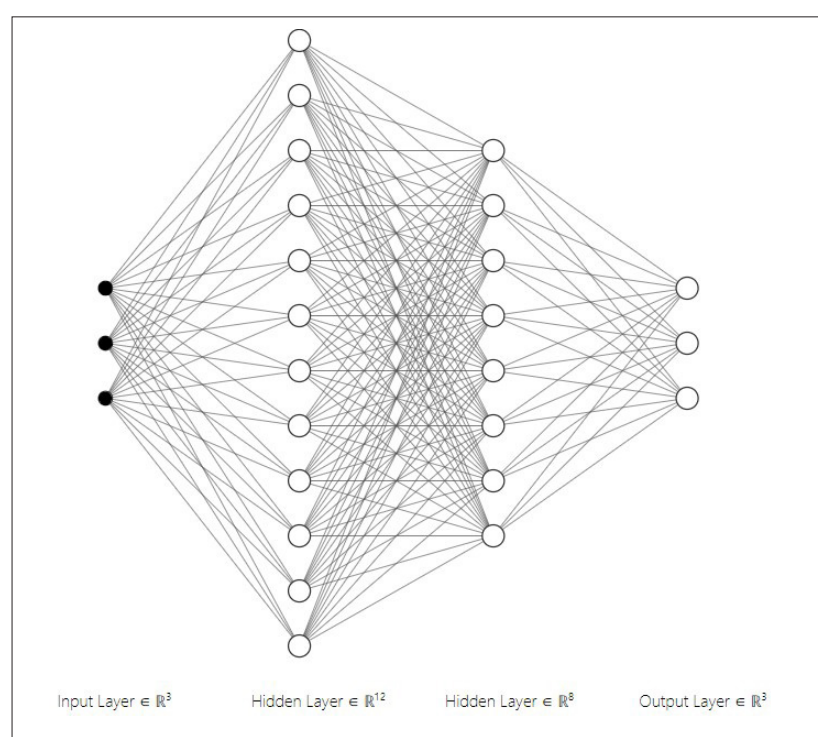

Figure 5. Architecture of multi-layer feed forward neural network 


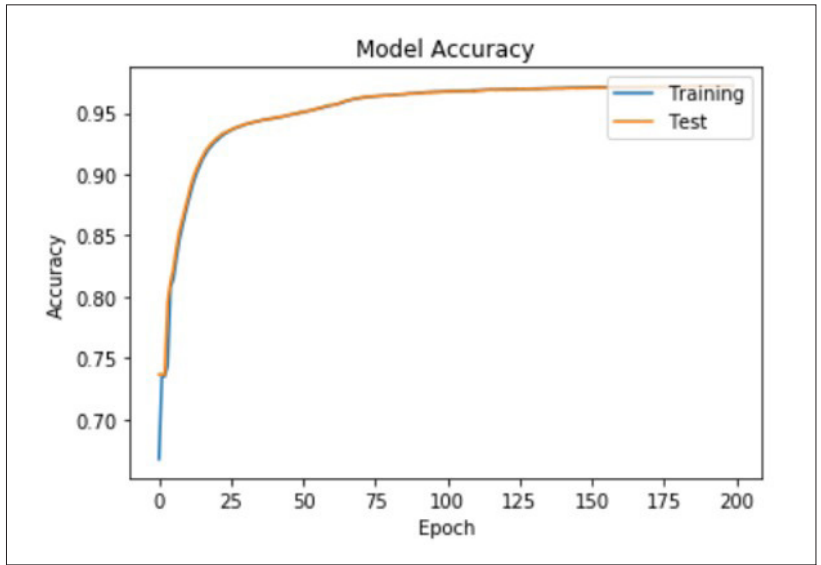

Figure 6. Model accuracy

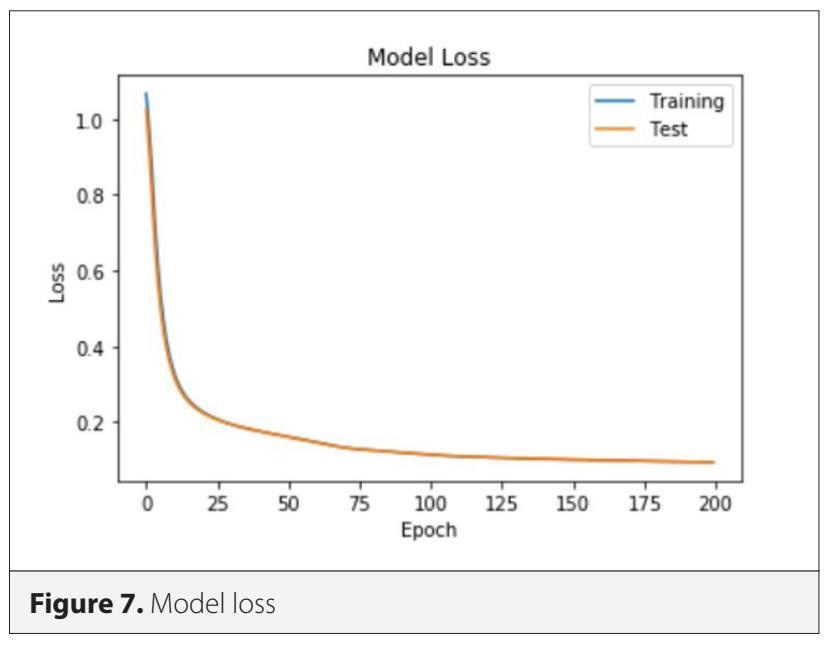

activation function in the hidden layers and the Softmax activation function is used in the output layer. The architecture of the ANN was developed using the Python language and the TensorFlow infrastructure was used [16] through the Keras [17] library and the Adam [18] optimizer.

The classification performance, cross entropy loss, or log loss of the feed forward neural network were measured as a probability value between 0 and 1 using the expression in Equation 1. As a result of the training, the accuracy rate of $\sim 98 \%$ and the loss function value is about 0.1 as shown in Figure 6 and Figure 7 respectively. As the predicted probability moves away from normal scenario conditions, the loss of cross entropy increases.

$L(y-\hat{y})=\sum_{i=1}^{n} y_{i} \log \hat{y}_{i}$

In hidden layers, ReLU activation function is used. ReLU is an activation function indicated by Equation 2, where $z$ is the value of 0 for each negative $z$ value and $z$ for each positive $z$ value.

$$
R(z)=\max _{z}(\mathbf{0}, z)
$$

The Softmax activation function is used in the output layer given by Equation 3. The Softmax function takes input vectors and normalizes the probability distribution function. After applying Softmax to the input vectors, the vector elements take a value between 0 and 1 .

$$
S\left(\Theta^{(i)}\right)=\frac{e^{\Theta^{(i)}}}{\sum_{j=0}^{k} e^{\Theta_{k}^{(i)}}}
$$

\begin{tabular}{|c|c|c|c|c|c|c|c|}
\hline \multicolumn{6}{|c|}{ FIRE EARIY WARNING SYSTEM FOR LARGE AREAS WITH ARTIFICIAL NEURAL NETWORK DECISION MECHANISM } & - & $x$ \\
\hline \multicolumn{8}{|c|}{$\begin{array}{l}\text { Please define the transmission distances (meters) and the latitude (degrees) } \\
\text { and longitude (degrees) initialization informations of the modules respectively } \\
\text { in order to determine the approximate positioning of the wireless communication } \\
\text { modules with google maps and to monitor all the modules on the web server. }\end{array}$} \\
\hline A0 Beacon & Distance: & 100 & Latitude: & 41.0282846 & Longitude: & 28.8898168 & \\
\hline A1 Sensor Node & Distance: & 100 & Latitude: & 41.0281758 & Longitude: & 28.8891246 & \\
\hline A2 Sensor Node & Distance: & 100 & Latitude: & 41.027931 & Longitude: & 28.8896785 & \\
\hline Bo Sensor Node & Distance: & 100 & Latitude: & 41.0290433 & Longitude: & 28.8905799 & \\
\hline B1 Sensor Node & Distance: & 100 & Latitude: & 41.0293692 & Longitude: & 28.8913502 & \\
\hline \multirow[t]{6}{*}{ B2 Sensor Node } & Distance: & 100 & Latitude: & 41.0298821 & Longitude: & 28.8919464 & \\
\hline & \multicolumn{4}{|c|}{ Define the entered values on Google Maps } & & & \\
\hline & \multicolumn{4}{|c|}{ Remember the previous entered values } & & & \\
\hline & \multicolumn{4}{|c|}{ Start the control and processing unit } & & & \\
\hline & \multicolumn{4}{|c|}{ Monitor status of all sensor nodes } & & & \\
\hline & & & Start Wet & & & & \\
\hline
\end{tabular}




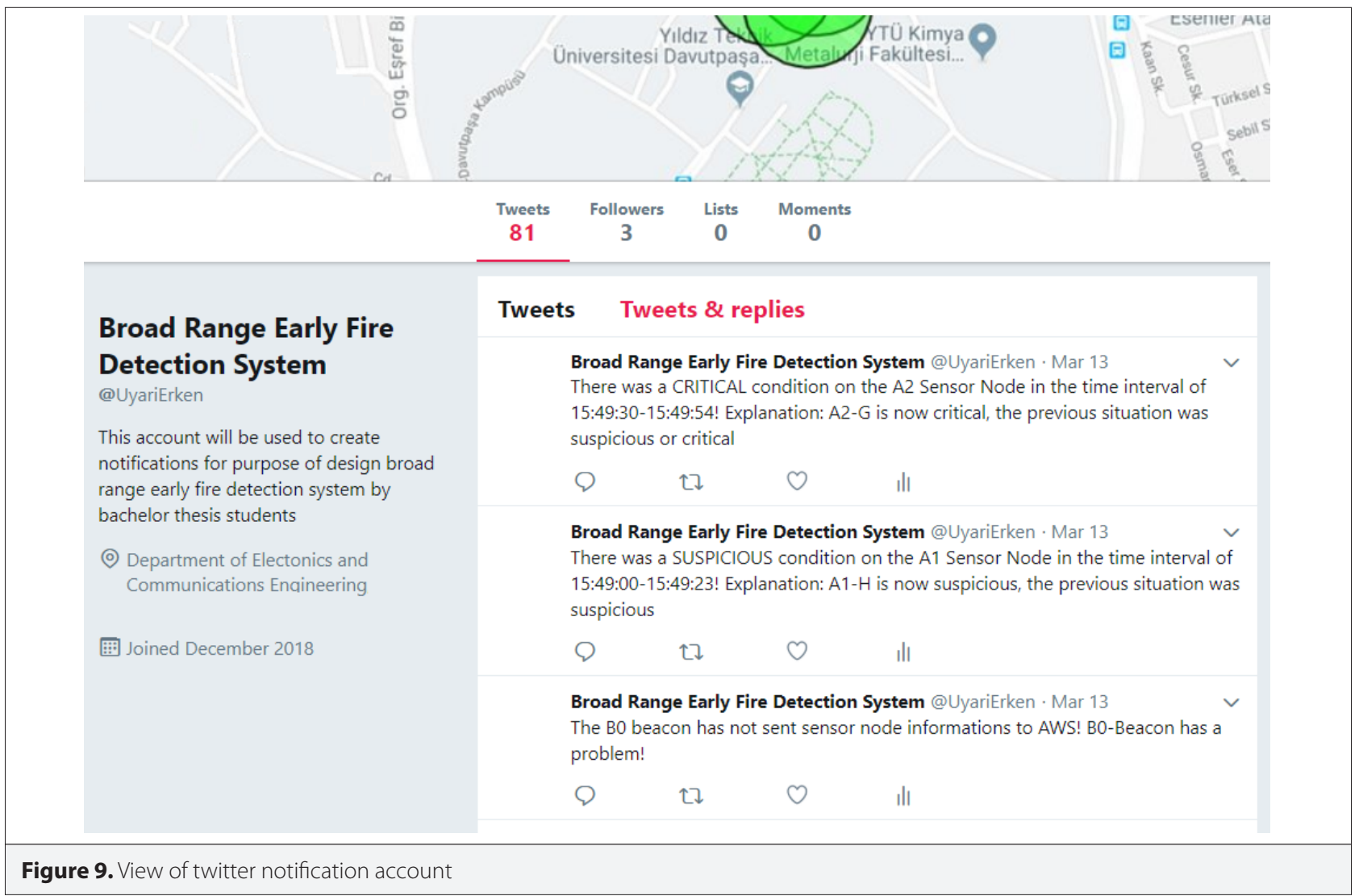

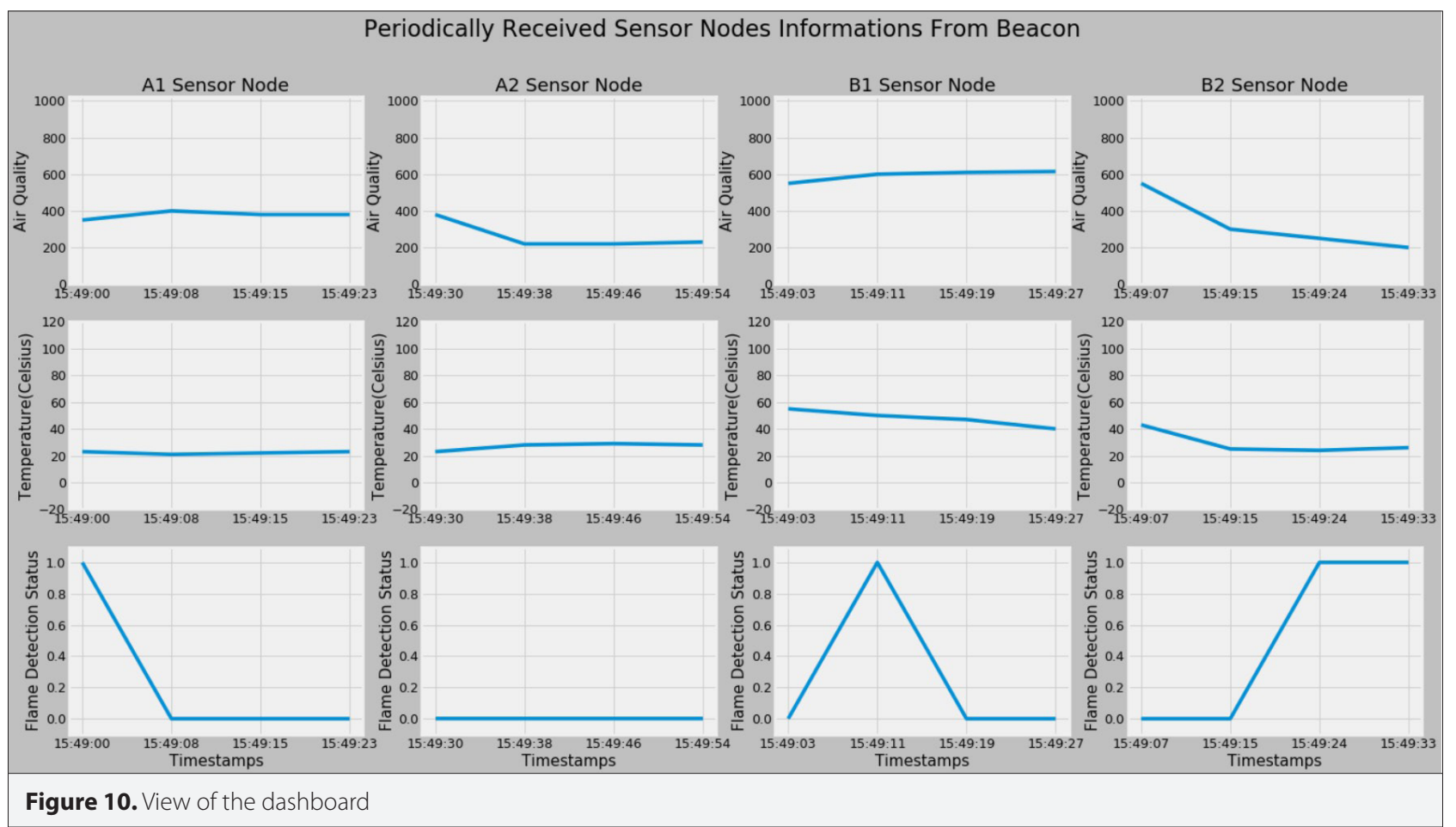

\section{Graphical User Interface Design}

The outputs produced by the Feed forward neural network in Beacon are transmitted to the server side for cloud data stor- age offered by Amazon Web Services (AWS). In this way, beacon and server communications are accomplished. The file containing sensor node information in csv format from Beacon periodically is sent to AWS. On server side, the server checks whether 


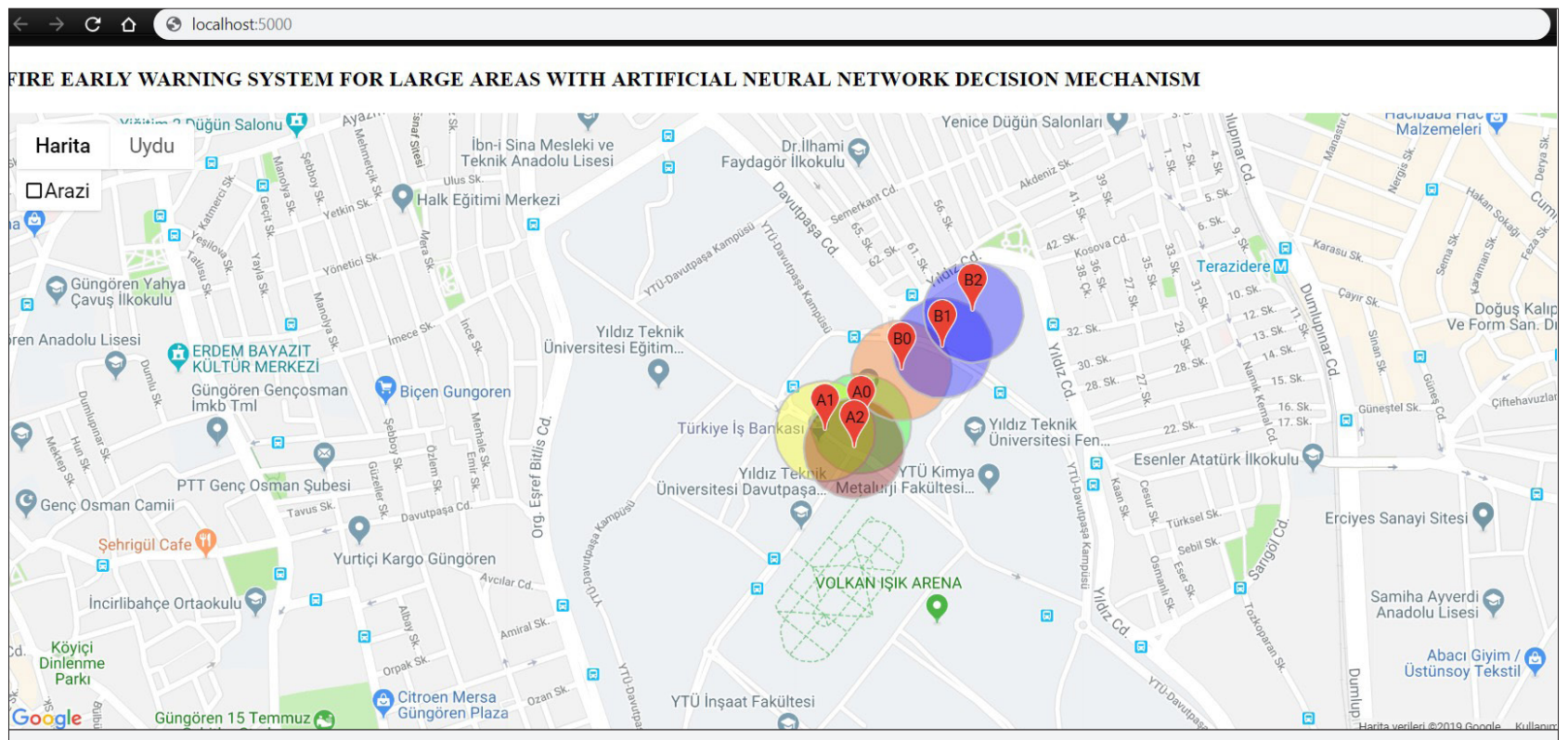

Figure 11. View of web server running in local machine

the file has been discarded. This file contains the sample sequence of the sensor node, the artificial neural network status assessment, the probability of the condition being evaluated, the temperature, the air quality, the flame sensor status information and the time label. The GUI provides an information about all important conditions for sensor nodes and beacons as seen in Figure 8.

The initial state of the sensor nodes and beacons is defined by using GUI to start the control and processing unit. In addition, launching the dashboard and the web server are also provided by this graphical user interface. In order to define initial information of the sensor nodes and beacons, the data transmission distance information of each selected modules must be known. The position information is defined for each module in the length and latitude in degrees via high-accuracy GPS module. Depending on the user's request, previous defined values can also be reminded. This initialization process integrated with Google Maps application programming interface. Mesh structure is visualized and provided approximate location detection for the modules. It is designed to be updated according to data received from AWS. The control and processing unit have many tasks. These tasks are control of sensor node information sent by beacons on AWS, checking periodically received module information with decision function, notification of the necessary situations to relevant authorities, visualization of dynamically updated mesh structure on the web server.

\section{Simulation and Results}

Simulation was performed using 2 Beacon and 4 sensor nodes. Position information of Beacons and sensor nodes are detected by a high-resolution external GPS. The circular radio range of the ZigBee module was calculated practically 100 meters. The circuit elements for the sensor node consume a total of 1.023 Watt power. Although the delay between one XBee to another XBee increases as the number of hop increases but, it is maximum 350 ms. Sensor informations such as temperature, air quality value and flame are transmitted with hexadecimal code with XBee modules. The sensor node information is periodically interpreted by the feed forward neural network. It is also periodically transmitted to the server side with instant time labeling and the decision process is executed.

\section{Informing the Outputs with Twitter}

With the support of the Twitter's application programming interface provided to the software developers, it is aimed to inform the relevant authorities instantly as shown in Figure 9.

\section{Designed Dashboard}

The designed control and processing unit ensures that the sensor node information transmitted to AWS is monitored periodically. Any information interpreted by feed forward neural network model can be tracked by the panel in Figure 10. The air quality and temperature values of each sensor node as well as the fire detection status can be monitored by the dashboard.

\section{Status Notification with Google Maps}

The location and changing status information of the beacon and sensor nodes can be monitored instantly using the Google Maps application programming interface shown in Figure 11 , The location information is determined by the MAC address of the data sent by the XBee modules. The current status of wireless module devices whose location is mapped via their MAC addresses are visualized by color codes on Google Maps. 


\section{Conclusion}

In this study, an early fire detection system has been implemented in a distributed mesh structure with wireless sensor networks. In general, this system consists of 3 main components: sensor node, beacon and server. Multi-layer feed forward neural networks were used as a decision maker. The outputs of the Neural Network were evaluated in the server side and the appropriate action was taken according to the situations. In the future study, it will be possible to have a wider coverage area of the system by increasing the number of sensor nodes. With this situation, it can be said that the mesh structure can be designed with more redundant paths. In addition, other sensor types suitable for early detection of fire can be integrated into the system. Thus, it is clear that the neural network with the other sensors will produce stronger output conditions of the new model to be created.

Peer-review: Externally peer-reviewed.

Conflict of Interest: The authors have no conflicts of interest to declare.

Financial Disclosure: The authors declared that the study has received no financial support.

\section{References}

1. P. Cheong, K. Chang, Y. Lai, S. KamHo, I. Sou, K. Tam, "A ZigBee-Based Wireless Sensor Network Node for Ultraviolet Detection of Flame", IEEE Transactions on industrial electronics, vol. 58, no. 11, pp. 5271-5277, November 2011. [CrossRef]

2. M. Hefeeda, M. Bagheri, Wireless Sensor Networks for Early Detection of Forest Fires. In Proceedings of the 2007 IEEE International Conf. on Mobile Adhoc and Sensor Systems, pp. 1-6, Pisa, Italy, October 2007. [CrossRef]

3. M. Bahrepour, N. Meratnia, M. Poel, Z. Taghikhaki, P. J. M. Havinga, "Distributed Event Detection in Wireless Sensor Networks for Disaster Management," in Intelligent Networking and Collaborative Systems, 2nd International Conference on, pp. 507-512, Thessaloniki, Greece, November 2010, [CrossRef]

4. P. U. S. Bolourchi, "Forest Fire Detection in Wireless Sensor Network Using Fuzzy Logic," Fifth International Conference on Computational Intelligence, Communication Systems and Networks, pp. 83-87, Spain, June 2013. [CrossRef]

5. J. Zhang, W. Li, Z. Yin, S. Liu, X. Guo, "Forest fire detection system based on wireless sensor network," in Proc 4th IEEE Conf Ind Electron Appl (ICIEA), pp. 520-523, Xi'an, China, May 2009. [CrossRef]
6. S. Mohapatra, P. M. Khilar, "Forest fire monitoring and detection of faulty nodes using wireless sensor network". In IEEE Region 10 Conference (TENCON) pp. 3232-3236, Singapore, 2016. [CrossRef]

7. S. Anand, R. K. Keetha Manjari,"FPGA implementation of artificial Neural Network for forest fire detection in wireless Sensor Network" in Computing and Communications Technologies (ICCCT), 2017 2nd International Conference on, Singapore, February 2017. [CrossRef]

8. H. Soliman, K. Sudan, A. Mishra, "A Smart Forest-Fire Early Detection Sensory System: Another Approach of Utilizing Wireless Sensor and Neural Networks" in IEEE Sensors Conference, Pittsburgh, PA, USA, November 1-4, 2010. [CrossRef]

9. A. Chehri, W. Farjow, H. T. Mouftah, X. Fernando, "Design of Wireless Sensor Network for Mine Safety Monitoring" in 24th Canadian Conference on Electrical and Computer Engineering, Ontario, Canada, May 8-11, 2011. [CrossRef]

10. A. K. Jain, A. Khare, K. K. Pandey, "Developing an Efficient Framework for Real Time Monitoring of Forest Fire Using Wireless Sensor Network" in Second IEEE International Conference on Parallel, Distributed and Grid Computing Conference, Solan, Himachal Pradesh, India, 6th to 8th December 2012. [CrossRef]

11. A. Bayo, D. Antolin, N. Medrano, B. Calvo, S. Celma, "Development of a Wireless Sensor Network System for Early Forest Fire Detection" in European Workshop on Smart Objects: Systems, Technologies and Applications, Ciudad, Spain, 15-16 June 2010.

12. Z. Chaczko, F. Ahmad, "Wirelee Sensor Network Based System for Fire Endangered Areas" in Third International Conference on Information Technology and Applications (ICITA'05), Sydney, NSW, Australia, 4-7 July 2005.

13. X. Yunjie, "Wireless sensor monitoring system of Canadian Poplar Forests based on Internet of Things", Artifcial Life and Robotics, pp: 1-9, 2019. [CrossRef]

14. Y.E. Aslan, I. Korpeoglu, O. Ulusoy, "A framework for use of wireless sensor networks in forest fire detectionand monitoring", pp: 614625, Computers, Environment and Urban Systems, No: 36, 2012 [CrossRef]

15. J. Yao, X. Zhang, A.T. Murray, "Location optimization of urbanfire stations: Access and service coverage" Computers, Environment and Urban Systems, pp: 184-190, No: 73, 2019 [CrossRef]

16. M. Abadi, A. Agarwal, P. Barham, E. Brevdo, Z. Chen, C. Citro, "TensorFlow: Large-scale machine learning on heterogeneous systems", Available from URL: http://download.tensorflow.org/ paper/whitepaper2015.pdf.

17. F. Chollet, (2015) Keras, GitHub. Retrieved from https://github. com/fchollet/keras.

18. D. Kingma, J. Ba, "Adam: A Method For Stochastic Optimization", The International Conference on Learning Representations, May 2015. 


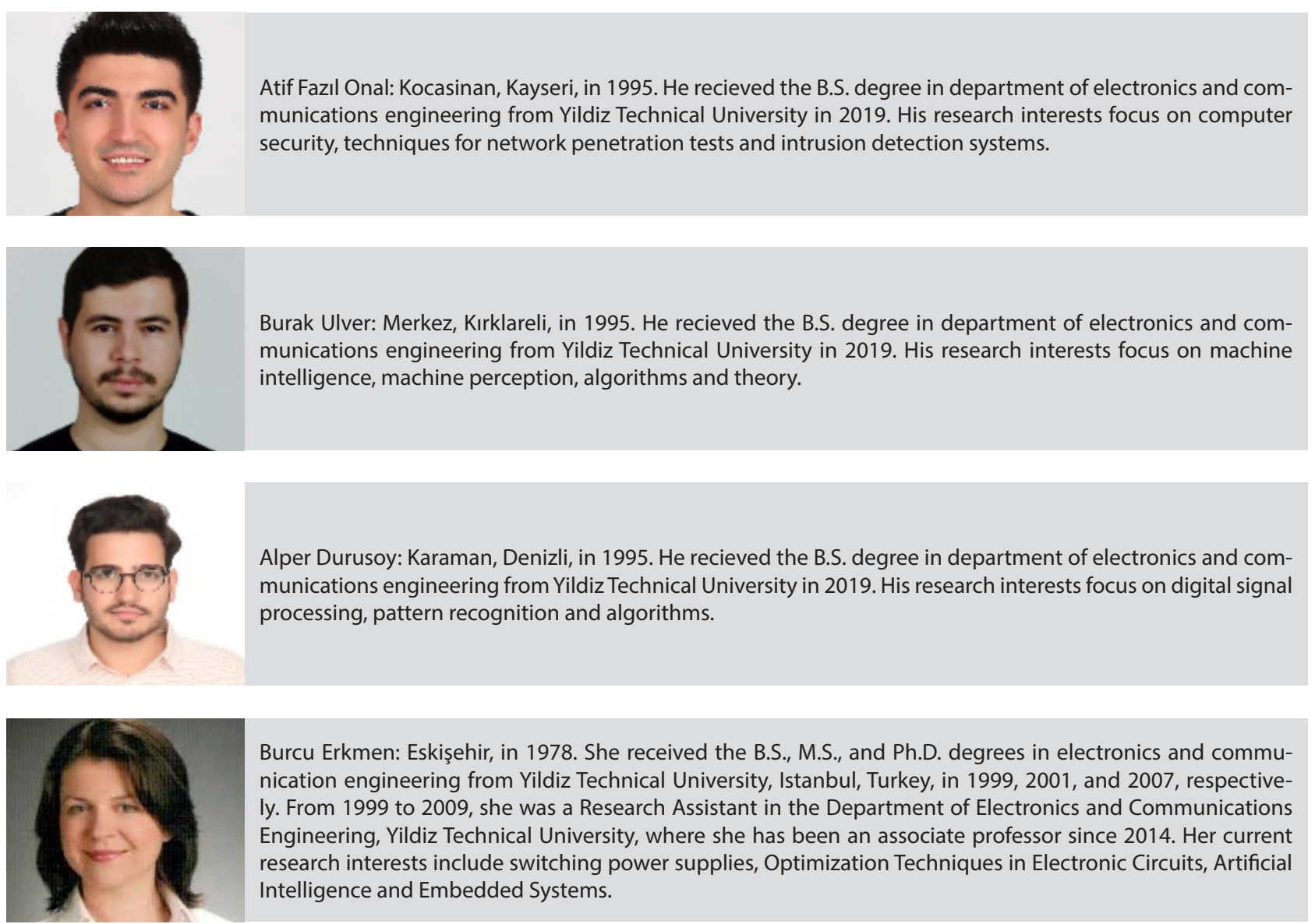

